\title{
Модуляция межподзонного поглощения света и межзонной фотолюминесценции в двойных квантовых ямах GaAs/AIGaAs в сильных продольных электрических полях
}

\author{
(C) Р.М. Балагула, М.Я. Винниченко, И.С. Махов, Д.А. Фирсов, Л.Е. Воробьев \\ Санкт-Петербургский политехнический университет Петра Великого, \\ 195251 Санкт-Петербург, Россия \\ E-mail: rmbal@spbstu.ru, mvin@spbstu.ru
}

(Получена 27 апреля 2016 г. Принята к печати 10 мая 2016 г.)

\begin{abstract}
Представлены результаты исследования влияния продольного электрического поля на спектры поглощения излучения среднего инфракрасного диапазона и на спектры межзонной фотолюминесценции в двойных туннельно-связанных квантовых ямах GaAs/AlGaAs. Полученные результаты объясняются перераспределением горячих электронов между ямами и изменением объемного заряда в структуре. Из анализа спектров модуляции межподзонного поглощения света и межзонной фотолюминесценции в сильных продольных электрических полях определена температура горячих носителей заряда.
\end{abstract}

\section{1. Введение}

Межподзонное поглощение света в структурах с квантовыми ямами (КЯ) активно исследуется достаточно давно. Особый интерес вызывают неравновесные оптические явления, сопровождающие, например, воздействие электрического поля на полупроводниковые структуры с КЯ. Ряд работ посвящен исследованию эффектов, возникающих в поперечном электрическом поле. Например, в работах [1-3] рассматривается влияние поперечного электрического поля, направленного вдоль оси роста структуры, и температуры решетки на спектр межподзонного поглощения света в туннельно-связанных квантовых ямах. Наблюдаемое изменение коэффициента межподзонного поглощения света объясняется перераспределением носителей заряда между уровнями размерного квантования и изменением энергетического спектра квантовых ям.

В продольном электрическом поле, приложенном в плоскости структуры, изменение межподзонного поглощения света в одиночных КЯ может быть связано с разогревом электронов в условиях непараболичности закона дисперсии подзон размерного квантования и учета обменного взаимодействия [4]. В квантовых ямах, имеющих уровень размерного квантования, близкий к потолку КЯ, разогрев электронов может привести к возникновению объемного заряда в структуре, что изменяет профиль потенциала и также меняет характеристики межподзонного поглощения $[5,6]$. Насколько нам известно, исследование влияния сильного продольного электрического поля на межподзонное поглощение света в туннельно-связанных КЯ ранее не проводилось. Исследованию данного эффекта, который может быть использован для быстрой модуляции инфракрасного излучения, посвящена настоящая работа.

Явление разогрева носителей заряда при высоких уровнях инжекции может влиять на характеристики полупроводниковых лазеров с КЯ. Разогрев носителей заряда сопутствует и другим физическим процессам, протекающим в полупроводниковых оптоэлектронных приборах в области больших токов накачки и больших уровней оптической инжекции. Одним из способов анализа степени разогрева носителей заряда является экспериментальное исследование спектров фотолюминесценции (ФЛ) при разных уровнях оптической накачки. Эта методика описана в ряде работ (см., например, $[7,8])$. В настоящей работе приводятся результаты исследования межзонной фотолюминесценции в туннельно-связанных КЯ при приложении продольного электрического поля.

\section{2. Методика эксперимента и обработки результатов}

Структура с туннельно-связанными КЯ для электрооптических исследований была выращена методом молекулярно-пучковой эпитаксии на полуизолирующей подложке GaAs. Она содержала 100 пар туннельносвязанных КЯ $\mathrm{GaAs} / \mathrm{Al}_{0.38} \mathrm{Ga}_{0.62} \mathrm{As}$ шириной 56 и $40 \AA$, разделенных туннельно-прозрачным барьером шириной $12 \AA$. Ширина туннельно-непрозрачного барьера между парами КЯ составляла $120 \AA$. В процессе роста КЯ были равномерно легированы кремнием с поверхностной концентрацией $N_{\mathrm{Si}}=6 \cdot 10^{11} \mathrm{~cm}^{-2}$. Расчет энергетического спектра электронов показал наличие трех уровней размерного квантования, два из которых локализованы в основном в широкой яме, а один - в узкой (см. рис. 1). При $T=77 \mathrm{~K}$ энергия межподзонных переходов между уровнями $e 1-e 3$ составляет 187 мэВ, энергия переходов $e 2-e 3=135$ мэВ.

На образец были нанесены индиевые омические контакты. После нанесения полоски индия отжигались в атмосфере азота. Образец плавно нагревался до температуры $300^{\circ} \mathrm{C}$ на протяжении 5 мин, затем в течение 5 мин происходил отжиг и далее температура плавно понижалась до комнатной. Грани образца были сточены 


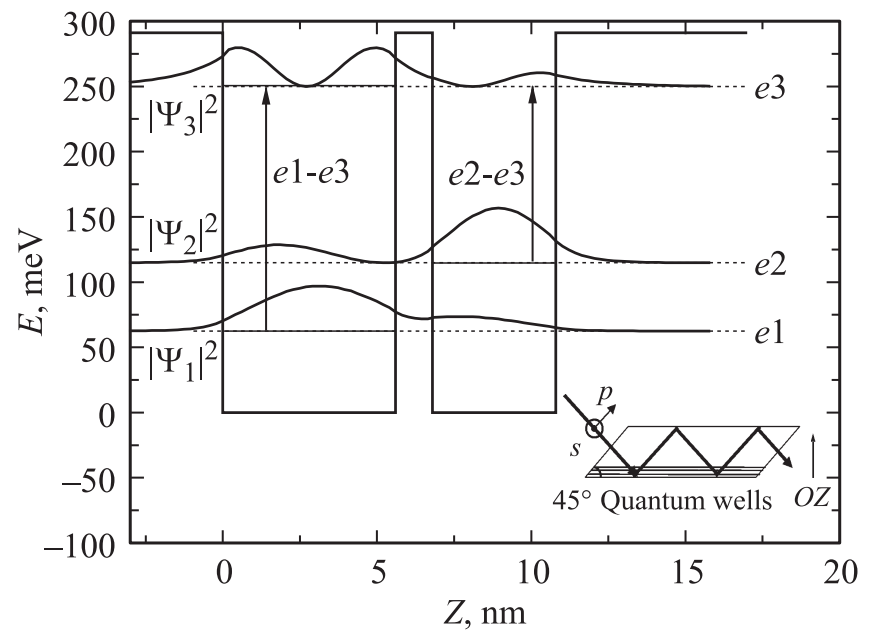

Рис. 1. Профиль дна зоны проводимости одного периода структуры при температуре $T=77 \mathrm{~K}$. Изображены рассчитанные положения уровней размерного квантования и квадраты модулей волновых функций электронов. Стрелками показаны межподзонные переходы $e 1-e 3$ и $e 2-e 3$. На вставке представлена схема образца в многопроходной геометрии.

под углом $45^{\circ}$, чтобы обеспечить многопроходную геометрию образца (см. вставку к рис. 1). Такая геометрия позволяет независимо исследовать поглощение света двух поляризаций. Для излучения $s$-поляризации вектор поляризации лежит в плоскости КЯ, такое излучение, согласно правилам отбора, не приводит к межподзонным переходам. Световая волна $p$-поляризации способна возбудить межподзонные оптические переходы, так как она имеет отличную от нуля проекцию вектора напряженности электрического поля на ось роста структуры $O Z$.

С целью подтверждения рассчитанного положения энергетических уровней были найдены спектры межзонной фотолюминесценции при различных температурах. Исследование межзонной ФЛ проводилось с помощью решеточного монохроматора Horiba FHR-640 в паре с CCD-камерой Symphony II. Для накачки использовался непрерывный твердотельный YAG-лазер с длиной волны излучения 532 нм. Влияние продольного электрического поля на межзонную фотолюминесценцию исследовалось на установке, собранной на базе решеточного монохроматора ИКС-21 и кремниевого фотодиода.

Спектры равновесного межподзонного поглощения света были получены в результате обработки спектров пропускания, найденных с использованием фурьеспектрометра Bruker Vertex 80v. В качестве источника излучения выступал глобар. Детектирование излучения осуществлялось охлаждаемым жидким азотом фотоприемником на основе твердого раствора кадмий-ртутьтеллур (КРТ). Образец помещался в криостат замкнутого цикла с рабочим диапазоном температур 4-320 K и окнами из $\mathrm{ZnSe}$.

Спектры изменения межподзонного поглощения в продольном электрическом поле были получены из спек- тров пропускания, измеренных на фурье-спектрометре в режиме пошагового сканирования. Во избежание перегрева образца использовалась импульсная методика приложения электрического поля (длительность импульсов 250 нс, скважность 50000-250000). Для получения спектров изменения межподзонного поглощения измерялись спектры изменения пропускания света p-поляризации двумя способами.

В первом случае фиксировалось изменение интенсивности света, прошедшего через образец, в продольном электрическом поле с помощью фотоприемника КРТ (АС канал) и импульсного синхронного детектора SR250, синхронизованного с генератором импульсов сильного поля. В этом методе на фурье-спектрометре измерялась интерферограмма, соответствующая непосредственному изменению в электрическом поле интенсивности прошедшего света $p$-поляризации $\Delta I_{p}$ относительно равновесной интенсивности. Отметим, что быстрое фурье-преобразование и классические фурьеспектрометры обычно работают только с положительными сигналами (стандартная методика фурьеспектроскопии не подразумевает сохранения информации о знаке сигнала). Результатом фурье-преобразования полученной интерферограммы является комплексный спектр $A(\omega)$, а не вещественный спектр изменения пропускания $\Delta I_{p}(\omega)$, получаемый при использовании традиционных дисперсионных спектрометров $[9,10]$ :

$$
A(\omega)=\left|\Delta I_{p}(\omega)\right| \exp \left[i\left(\varphi_{0}(\omega)+\Delta \varphi(\omega)\right)\right]
$$

где экспоненциальный множитель содержит фазовую ошибку $\varphi_{0}(\omega)$ и фазовый сдвиг $\Delta \varphi(\omega)$, соответствующий изменению знака сигнала. Очевидно, что положительное и отрицательное изменения сигнала будут соответствовать $\Delta \varphi=0$ и $\Delta \varphi=\pi$. Для восстановления истинного амплитудного спектра $\Delta I_{p}(\omega)$ из комплексной функции $A(\omega)$ необходимо провести процедуру фазовой коррекции. На сегодняшний день существует ряд методов фазовой коррекции: домножение спектра $A(\omega)$ на комплексно-сопряженную функцию, метод Формана, метод Мерца и др. Наименее чувствительным к шумам является метод мультипликативной фазовой коррекции или метод Мерца [11], который заключается в нахождении действительной части от произведения $A(\omega)$ и инвертированного фазового множителя:

$$
\Delta I_{p}(\omega)=\operatorname{Re}\left[A(\omega) \exp \left({ }_{i} \varphi_{0}(\omega)\right)\right]
$$

Фазовая ошибка $\varphi_{0}(\omega)$ является параметром эксперимента и установки, в случае положительного амплитудного спектра фазовая ошибка не должна влиять на амплитудный спектр $\Delta I_{p}(\omega)$. Для измерения фазовой ошибки $\varphi_{0}(\omega)$ использовался выход фотоприемника по постоянному току (DC канал), который регистрировал интерферограмму и фазу спектра поглощения в отсутствие электрического поля. В этом случае спектр является заведомо положительным и не имеет резких 
скачков фазы на $\pi$. Так как фазовая ошибка определяется в первую очередь характеристиками интерферометра [12] и параметрами эксперимента, одновременное измерение $\varphi_{0}(\omega)$ и $A(\omega)$ позволило нам провести фазовую коррекцию и получить истинный спектр изменения пропускания $\Delta I_{p}$.

Во втором случае изменение межподзонного поглощения в электрическом поле $F$ регистрировалось путем измерения спектров пропускания с временны́м разрешением и вычитанием из спектров в электрическом поле $I_{p}(\omega, t, F)$ спектров без него $I_{p}(\omega, t, F=0)$ :

$$
\Delta I_{p}(\omega, t, F)=I_{p}(\omega, t, F)-I_{p}(\omega, t, F=0) .
$$

Спектры пропускания определялись с временным разрешением в 25 нс. Измерение пропускания начиналось за 1 мкс до начала действия импульса поля и заканчивалось через 25 мкс после окончания импульса поля. Это позволяло оценить вклад нагрева решетки и разогрева электронов в изменение пропускания.

\section{3. Результаты и обсуждение}

Для подтверждения рассчитанного энергетического спектра носителей заряда в КЯ были измерены спектры межзонной ФЛ в широком диапазоне мощностей оптической накачки и температур $(77-300 \mathrm{~K})$. При температуре жидкого азота (штриховая линия на рис. 2) наблюдается только пик излучения, связанный с переходами $e 1-h h 1$. Это связано с тем, что при низкой температуре носители заряда в основном локализованы на уровнях $e 1$ и $h h 1:$ например, при $T=77 \mathrm{~K}$ концентрация электронов на уровне $e 1$ на 3 порядка выше, чем на уровне $e 2$. При комнатной температуре концентрации на уровнях $e 1$ и $e 2$ отличаются лишь на порядок, в результате в спектре дополнительно наблюдались особенности, соответствующие переходам с участием возбужденных электронных состояний и состояний легких и тяжелых дырок: $e 1-h h 1, e 2-h h 2, e 1-l h 1$ (сплошная линия на рис. 2). Температурный сдвиг пиков ФЛ объясняется изменением ширины запрещенной зоны материалов барьеров и КЯ. Положение всех пиков удовлетворительно согласуется с результатами расчета энергетического спектра.

Для получения спектров межподзонного поглощения были измерены спектры пропускания света $p$ - и $s$-поляризации. Из закона Бугера-Ламберта-Бера следует, что

$$
\ln \left(I_{p} / I_{s}\right)=-\alpha L
$$

где $I_{p}, I_{s}$ - интенсивности прошедшего света $p$ - и $s$-поляризации соответственно, $\alpha-$ коэффициент межподзонного поглощения света в квантовых ямах, $L-$ длина оптического пути в структуре. На рис. 3 представлены спектры пропускания света $p$ - и $s$-поляризации при $T=77 \mathrm{~K}$. Положение линии поглощения в области энергий кванта 180-200 мэВ для излучения $p$-поляризации

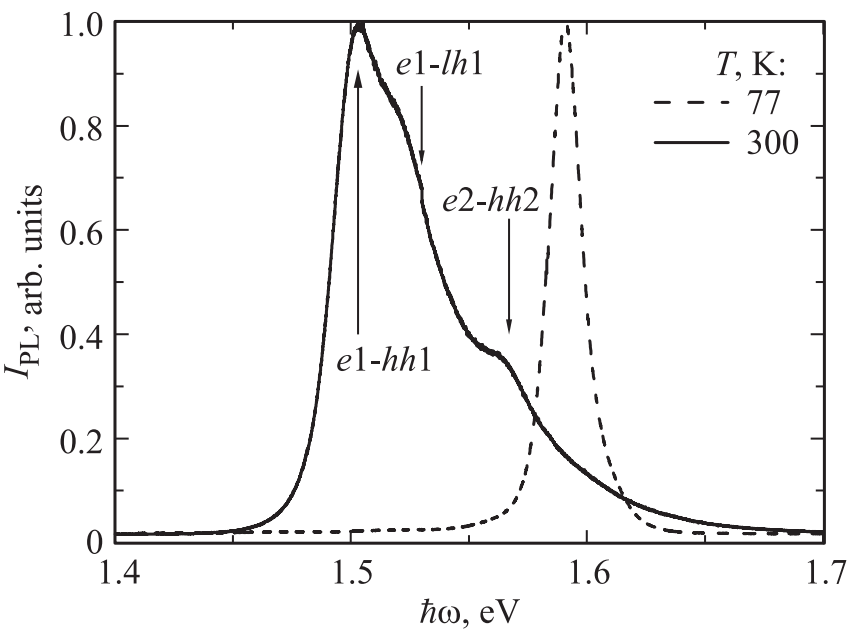

Рис. 2. Нормированные спектры межзонной фотолюминесценции при температурах $T=77 \mathrm{~K}$ (штриховая линия) и $T=300 \mathrm{~K}$ (сплошная линия). Стрелками показаны рассчитанные энергии межзонных переходов $e 1-h h 1, e 2-h h 2$ и $e 1-l h 1$ при $T=300 \mathrm{~K}$.

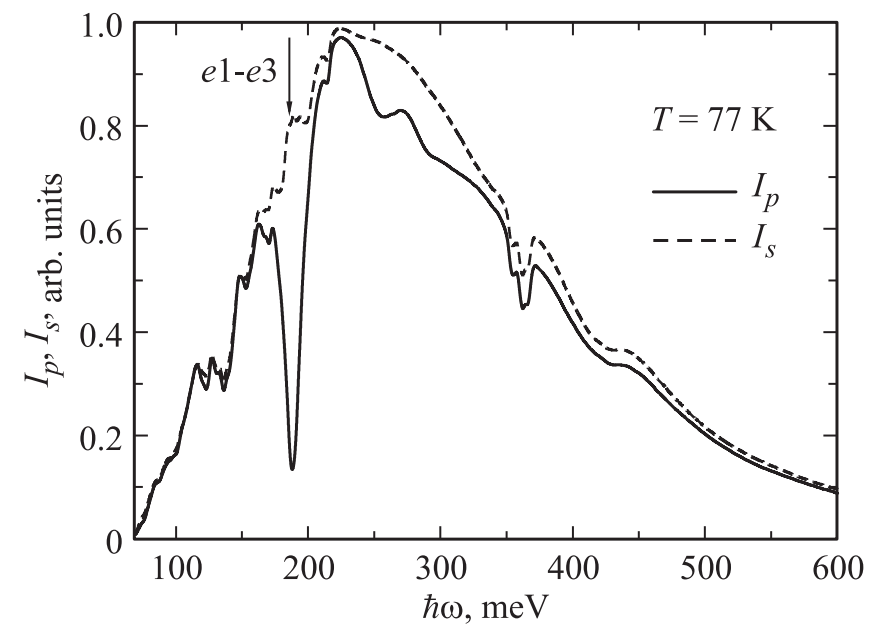

Рис. 3. Нормированные спектры интенсивности света двух поляризаций, прошедшего через образец, при температуре $T=77 \mathrm{~K}$. Стрелкой показано рассчитанное значение энергии переходов $e 1-e 3$.

(сплошная линия на рис. 3) хорошо согласуется с теоретически рассчитанным значением энергии перехода $e 1-e 3$ (указано на рис. 3 стрелкой). В соответствии с правилами отбора для межподзонных переходов электронов в КЯ поглощается только свет, имеющий компоненту поляризации, направленную перпендикулярно слоям с КЯ, т. е. свет р-поляризации.

Явление полного внутреннего отражения, используемое в многопроходной геометрии эксперимента, может привести к возникновению стоячей световой волны в образце [13]. Наличие на поверхности исследуемого образца сильно легированного слоя приводит к тому, что эта стоячая волна будет иметь узел на поверх- 


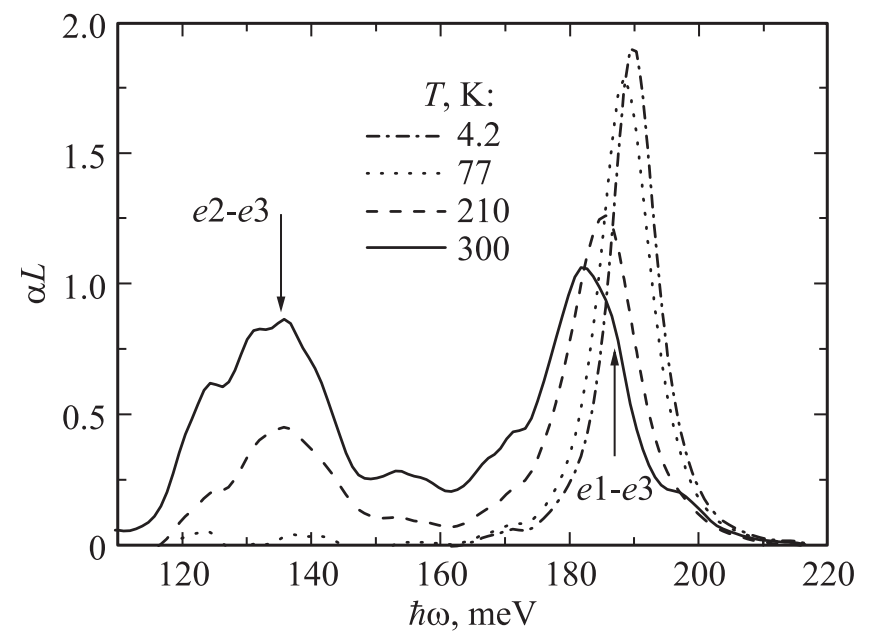

Pис. 4. Спектры межподзонного поглощения света при разных температурах решетки. Стрелками показаны рассчитанные энергии переходов электронов при $T=77 \mathrm{~K}$.

ности для света $s$-поляризации и пучность для света p-поляризации. Однако в используемой структуре этим эффектом можно пренебречь, поскольку она содержит 10 пар квантовых ям, и толщина активного слоя, дающего вклад в поглощение на межподзонных переходах электронов, составляет несколько микрон, что соответствует нескольким периодам стоячей волны. На таком расстоянии интенсивность излучения в КЯ, находящихся в узлах и пучностях стоячей волны, усредняется [13].

Спектры поглощения для широкого диапазона температур решетки были получены из спектров пропускания с использованием соотношения (4) (см. рис. 4). Видно, что при низких температурах в основном проявляются оптические переходы $e 1-e 3$ (оптические переходы $e 1-e 2$ находятся вне области спектральной чувствительности фотодетектора), т. е. электроны локализованы преимущественно на уровне $e 1$. При повышении температуры электроны начинают заполнять вышележащий энергетический уровень, что приводит к появлению пика поглощения, связанного с переходами $e 2-e 3$. Наблюдаемое смещение пика поглощения $e 1-e 3$ в длинноволновую область в первую очередь связано с температурной зависимостью ширины запрещенной зоны материалов барьера и КЯ и согласуется с расчетом энергетических уровней электронов. Также стоит отметить, что уровень $e 1$,генетически“ связан с широкой КЯ, в то время как уровень 22 связан в большей степени с узкой КЯ. Перераспределение носителей заряда между уровнями, связанными с разными квантовыми ямами, приводит к изменению объемного заряда в структуре и возникновению электрического поля между узкой и широкой квантовыми ямами, что приводит к изменению профиля потенциала и также дает вклад в смещение пика поглощения.

Методика измерения спектров модуляции пропускания в продольном электрическом поле была описана вы- ше в разделе „Методика эксперимента“. Для получения спектров модуляции межподзонного поглощения $\Delta \alpha$ из спектров модуляции пропускания необходимо воспользоваться соотношением, полученным из закона БугераЛамберта-Бера:

$$
\Delta \alpha L=-\ln \left(\Delta I_{p} / I_{p}+1\right) .
$$

Измерения спектров модуляции пропускания вторым методом, т. е. путем вычитания из интенсивности излучения, прошедшего через образец, в электрическом поле интенсивности излучения в отсутствие поля, в соответствии с формулой (3), проводились преимущественно для подтверждения результатов фазовой коррекции, которая выполнялась в процессе обработки данных о пропускании, полученных первым методом. Результаты измерений пропускания вторым методом были получены в виде трехмерных зависимостей интенсивности прошедшего через образец света $p$-поляризации $I_{p}$ от времени и энергии кванта. Зависимости интенсивности $I_{p}$ от времени представлены на рис. 5 для двух значений энергии кванта, соответствующих оптическим переходам $e 1-e 3(\hbar \omega=187$ мэВ, сплошная линия на рис. 5) и $e 2-e 3(\hbar \omega=135$ мэВ, штриховая линия на рис. 5), при температуре решетки $T=77 \mathrm{~K}$ и при значении напряженности приложенного продольного электрического поля $F=695 \mathrm{~B} / \mathrm{cm}$. Из данных, представленных на рис. 5, видно, что при приложении электрического поля наблюдается рост поглощения, связанного с оптическими переходами $e 2-e 3$, и уменьшение поглощения, связанного с переходами $e 1-e 3$. Через несколько микросекунд после окончания импульса электрического поля величина интенсивности $I_{p}$ достигает равновесного значения, что свидетельствует о пренебрежимо малом разогреве решетки. Каждый спектр модуляции пропускания был получен, согласно (3), как разность между

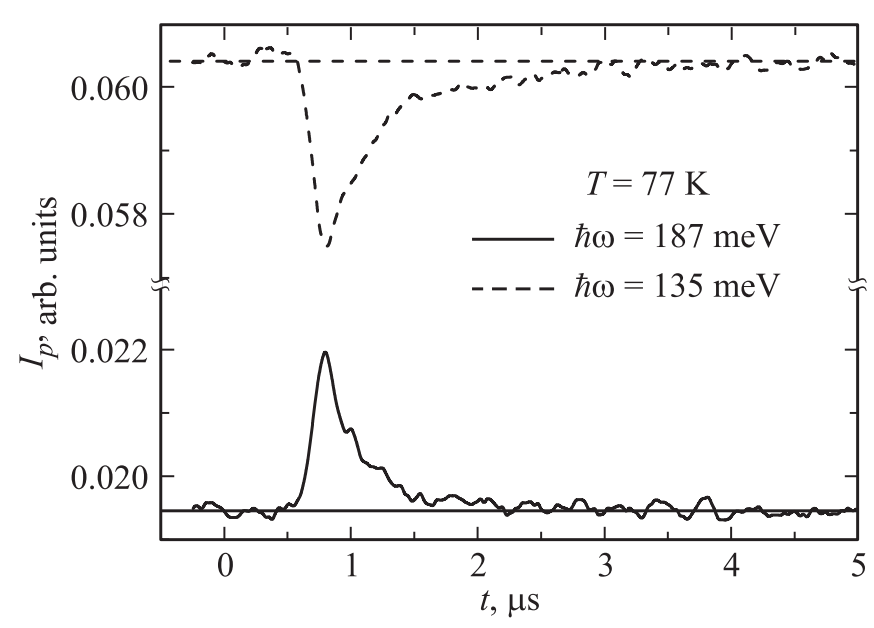

Рис. 5. Зависимости интенсивности света р-поляризации, прошедшего через образец, от времени при $T=77 \mathrm{~K}$, $F=695 \mathrm{~B} /$ см при энергиях кванта $\hbar \omega=187$ мэВ (сплошная кривая) и $\hbar \omega=135$ мэВ (штриховая кривая), которые соответствуют межподзонным переходам $e 1-e 3$ и $e 2-e 3$. 


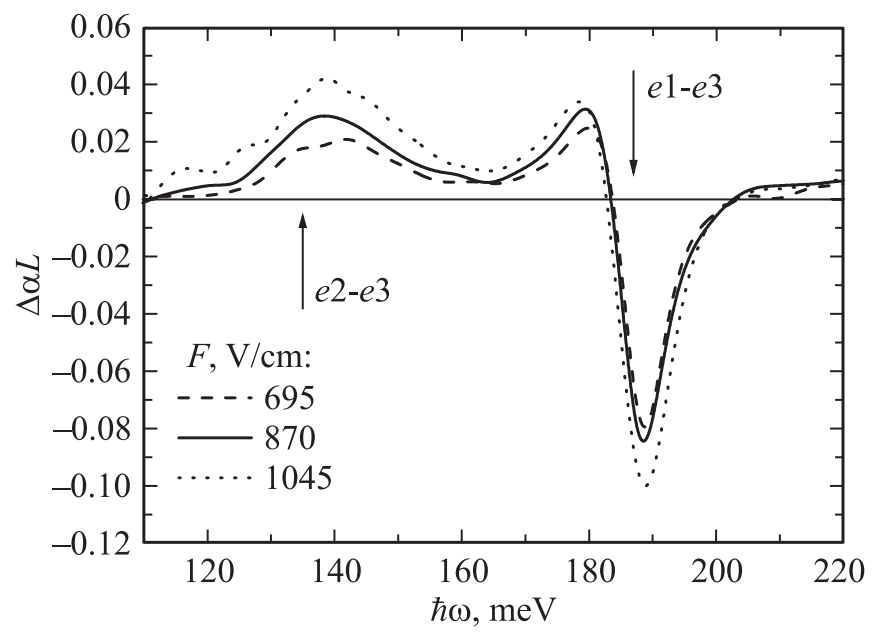

Рис. 6. Спектры модуляции межподзонного поглощения продольным электрическим полем при температуре $T=77 \mathrm{~K}$. Стрелками показаны рассчитанные энергии межподзонных переходов электронов.

величиной пропускания при приложении электрического поля в момент наибольшего изменения интенсивности прошедшего света $(t=0.8$ мкс на рис. 5) и величиной пропускания без электрического поля. Спектры модуляции пропускания структуры при приложении электрического поля, полученные обоими методами, идентичны, что подтверждает корректность выбранной процедуры фазовой коррекции.

На рис. 6 представлены спектры модуляции поглощения света под действием продольного электрического поля, полученные из спектров модуляции пропускания согласно формуле (5). Они были найдены для различных значений продольного электрического поля при темпеpaтуре $T=77 \mathrm{~K}$. Наблюдаемое увеличение поглощения в области переходов $e 2-e 3$ может быть объяснено разогревом носителей заряда на уровне $e 1$ продольным электрическим полем, в результате чего неравновесные электроны занимают вышележащие электронные состояния. Увеличение числа носителей заряда в подзоне $e 2$ приводит к росту межподзонного поглощения $e 2-e 3$ $(\hbar \omega=135$ мэВ). Уменьшение поглощения в области переходов $e 1-e 3(\hbar \omega=187$ мэВ) соответствует уменьшению концентрации носителей заряда в подзоне $e 1$ и сдвигу пика поглощения $e 1-e 3$ в область меньших энергий под воздействием продольного электрического поля.

Сдвиг линии межподзонного поглощения при переходах электронов $e 1-e 3$ в электрическом поле объясняется сдвигом уровней энергии, связанным с изменением объемного заряда в структуре из-за перераспределения неравновесных горячих электронов в реальном пространстве между двумя квантовыми ямами. Сравнение изменения поглощения в максимуме пика $\Delta \alpha L$ в электрическом поле с изменением поглощения при увеличении температуры решетки без электрического поля позволило оценить электронную температуру в электрическом поле: $T_{e}=103 \mathrm{~K}$ в поле $F=1045 \mathrm{~B} / \mathrm{cm}$.

Экспериментально были также получены спектры модуляции интенсивности межзонной фотолюминесценции в продольном электрическом поле при $T=77 \mathrm{~K}$ (см. рис. 7). Температура горячих носителей заряда в используемых электрических полях не была достаточно высока, чтобы заселение уровня $e 2$ проявлялось на спектрах изменения ФЛ. В эксперименте наблюдалось изменение интенсивности ФЛ в электрическом поле для межзонных переходов $e 1-h h 1$, которое мы связываем с разогревом электронов и дырок. Сравнение изменения спектра ФЛ при изменении температуры решетки и при приложении электрического поля при $T=77 \mathrm{~K}$ позволяет найти температуру горячих носителей заряда в электрическом поле. Изменение спектров ФЛ наблюдалось при достаточно высоких уровнях межзонной оптической накачки, поэтому можно считать, что вследствие интенсивного электронно-дырочного рассеяния температуры электронов и дырок примерно равны.

Запишем число квантов спонтанного межзонного излучения, испущенных в единице объема в единицу времени в интервале частот от $\omega$ до $\omega+d \omega$, для межзонных переходов $e 1-h h 1[14]$ :

$$
d q_{s p}(\omega) \sim \omega f_{e 1}(\omega) f_{h h 1}(\omega) d \omega,
$$

где $f_{e 1}, f_{h h 1}-$ функции распределения неравновесных электронов и дырок по энергиям в подзонах $e 1$ и $h h 1$ соответственно. Спектральная плотность интенсивности спонтанного излучения $I_{P L}$ пропорциональна величине $d q_{s p} / d \omega$. Используя (6) и функцию распределения Больцмана, легко получить [7,8,14]:

$$
I_{P L}(\omega) \sim \omega \cdot \exp \left[-\left(\hbar \omega-E_{g}^{*}\right) / k T_{e}\right],
$$

где $k-$ постоянная Больцмана, $T_{e}-$ температура горячих носителей заряда, $E_{g}^{*}$ - эффективная ширина

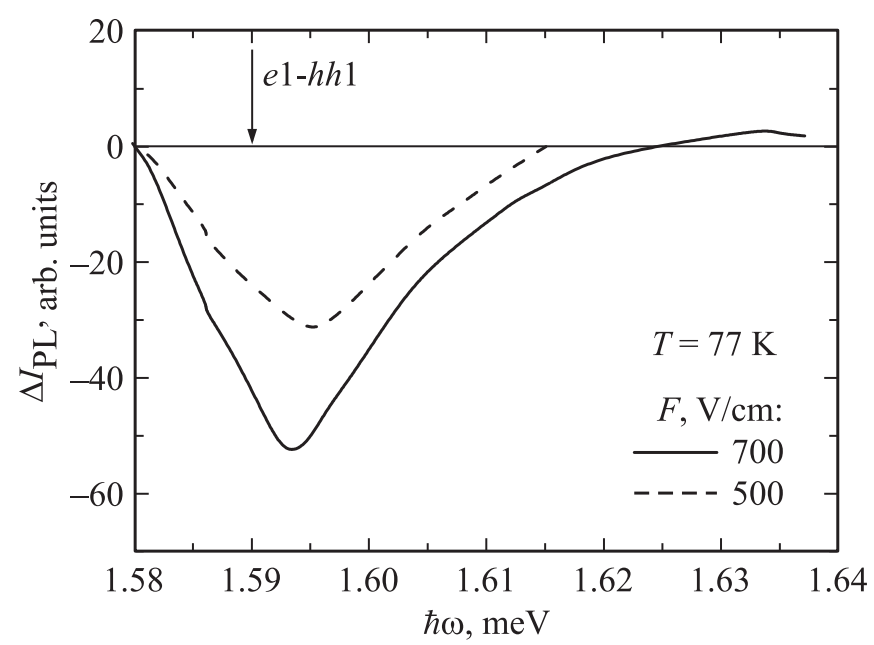

Pис. 7. Спектры модуляции межзонной фотолюминесценции при температуре решетки $T=77 \mathrm{~K}$ для двух значений продольного электрического поля. Стрелкой показано рассчитанное значение энергии межзонных переходов $e 1-h h 1$. 
запрещенной зоны, соответствующая энергии переходов $e 1-h h 1 \quad\left(E_{g}^{*}=E_{g}+E(e 1)+E(h h 1)\right)$. Зависимость (7) позволяет по коротковолновому краю спектра равновесной ФЛ определить зависимость $T_{e}$ от интенсивности оптической накачки. Для определения температуры горячих носителей заряда в продольном электрическом поле необходимо определить энергию кванта $\hbar \omega_{0}$ на экспериментальном спектре модуляции ФЛ электрическим полем, при которой $\Delta I_{P L}=0$, что соответствует $d q_{s p} / d T_{e}=0$. Дифференцируя соотношение (6) и используя функцию распределения Больцмана, можно найти температуру неравновесных носителей заряда:

$$
T_{e} \approx \frac{\hbar \omega_{0}-E_{g}^{*}}{2 k} .
$$

Таким образом, температура носителей заряда может быть определена из анализа изменения спектров ФЛ в сильном продольном электрическом поле с помощью соотношения (8). В поле $F=700 \mathrm{~B} /$ см увеличение электронной температуры, вызванное электрическим полем, оказалось равным примерно $30 \mathrm{~K}$. Это значение оказалось близким к температуре, полученной из анализа изменения спектров межподзонного поглощения света в сильном электрическом поле.

\section{4. Заключение}

В работе представлены результаты исследования влияния продольного электрического поля на спектры межподзонного поглощения излучения среднего инфракрасного диапазона и на спектры межзонной фотолюминесценции в туннельно-связанных квантовых ямах $\mathrm{GaAs} / \mathrm{AlGaAs}$. Приведены детали фазовой коррекции интерферограмм знакопеременного сигнала модуляции пропускания. Наблюдаемые особенности в измеренных спектрах равновесного межподзонного поглощения при различных температурах решетки и межподзонного поглощения в продольном электрическом поле объяснены перераспределением носителей заряда между квантовыми ямами и изменением объемного заряда. Сопоставление величины изменения поглощения в электрическом поле с изменением поглощения при увеличении температуры решетки позволило оценить электронную температуру в электрическом поле. Из спектров модуляции фотолюминесценции в электрическом поле определена температура неравновесных носителей заряда, близкая к температуре, полученной из анализа спектров изменения межподзонного поглощения света в сильном электрическом поле.

Работа выполнена при финансовой поддержке РФФИ (гранты № 14-02-00336, 16-32-60085), гранта Президента РФ для молодых кандидатов наук МК-4616.2016.2 и Министерства образования и науки России (государственное задание).

\section{Список литературы}

[1] E. Dupont, D. Delacourt, V. Berger, N. Vodjdani, N. Papuchon. Appl. Phys. Lett., 62, 1907 (1993).

[2] D.A. Firsov, L.E. Vorobjev, M.Ya. Vinnichenko, R.M. Balagula, M.M. Kulagina, A.P. Vasil'iev. Semiconductors, 49 (11), 1425 (2015).

[3] D.A. Rybalko, M.Ya. Vinnichenko, L.E. Vorobjev, D.A. Firsov, R.M. Balagula, V.Yu. Panevin, M.M. Kulagina, A.P. Vasil'iev. J. Phys.: Conf. Ser., 541, 012081 (2014).

[4] L.E. Vorob'ev, D.A. Firsov, V.A. Shalygin, I.I. Saidashev. JETP Lett., 65 (7), 549 (1997).

[5] E. Towe, D. Sun, L.E. Vorobjev, S.N. Danilov, D.A. Firsov, E.A. Zibik. Superlat. Microstr., 17 (2), 129 (1995).

[6] L.E. Vorobjev, E.A. Zibik, Yu.V. Kochegarov, S.N. Danilov, D.A. Firsov, E. Towe, D. Sun, A.A. Toropov, T.V. Shubina. Semiconductors, 29 (6), 588 (1995).

[7] J. Shah. Spectroscopy of nonequilibrium electrons and phonons ed. by C.V. Shank, B.P. Zakharchenya (AmsterdamLondon-N.Y.-Tokyo, Elsevier, 1992) chap. 2, p. 57.

[8] L.E. Vorobjev, M.Ya. Vinnichenko, D.A. Firsov, V.L. Zerova, V.Yu. Panevin, A.N. Sofronov, P. Thumrongsilapa, V.M. Ustinov, A.E. Zhukov, A.P. Vasiljev, L. Shterengas, G. Kipshidze, T. Hosoda, G. Belenky. Semiconductors, $44(11), 1402$ (2010).

[9] D.D. Firsov, O.S. Komkov. Techn. Phys. Lett., 39(12), 1071 (2013).

[10] А.С. Крылов, А.Н. Втюрин, Ю.В. Герасимова. Обработка данных инфракрасной фурье-спектроскопии (Красноярск, Ин-т физики СО РАН, 2005).

[11] L. Mertz. Infr. Phys., 7, 17 (1967).

[12] P.R. Griffiths, J.A. De Haseth. Fourier Transform Infrared Spectrometry (Hoboken, New Jersey, Wiley \& Sons, 2007).

[13] H. Schneider, C. Schönbein, M. Walther, P. Koidl, G. Weimann. Appl. Phys. Lett., 74 (1), 16 (1999).

[14] L.E. Vorob'ev, V.L. Zerova, K.S. Borshchev, Z.N. Sokolova, I.S. Tarasov, G. Belenky. Semiconductors, 42 (6), 737 (2008).

Редактор Г.А. Оганесян

\section{Modulation of intersubband light absorption and interband photoluminescence in double GaAs/AIGaAs quantum wells under lateral electric field}

\section{R.M. Balagula, M.Ya. Vinnichenko, I.S. Makhov, D.A. Firsov, L.E. Vorobjev}

Peter the Great St. Petersburg Polytechnic University, 195251 St. Petersburg, Russia

Abstract Lateral electric field influence on mid-infrared absorption and interband photoluminescence spectra in double tunnel-coupled GaAs/AlGaAs quantum wells was investigated. The obtained results can be attributed to electron redistribution between quantum wells in real space and space charge variation in the structure. Hot carrier temperature was estimated by means of analysis of intersubband absorption and interband photoluminescence modulation spectra under high lateral electric field. 